\title{
Mutation of ten-eleven translocation-2 is associated with increased risk of autoimmune disease in patients with myelodysplastic syndrome
}

Yoon-Jeong Oh, ${ }^{1,}$, Dong-Yeop Shin ${ }^{2,3,}$, Sang Mee Hwang ${ }^{4}$, Sung-Min Kim ${ }^{5}$, Kyongok Im ${ }^{5}$, Hee Sue Park ${ }^{6}$, Jung-Ah Kim ${ }^{6}$, Yeong Wook Song ${ }^{7}$, Ana Márquez ${ }^{8}$, Javier Martín ${ }^{8}$, Dong-Soon Lee ${ }^{5,6}$, and Jin Kyun Park

\author{
${ }^{1}$ Division of Rheumatology, \\ Department of Internal Medicine, \\ Kangwon National University \\ School of Medicine, Chuncheon; \\ ${ }^{2}$ Division of Hematology and \\ Medical Oncology, Department of \\ Internal Medicine, Seoul National \\ University College of Medicine, \\ Seoul; ${ }^{3}$ Biomedical Research \\ Institute, Seoul National University \\ Hospital, Seoul; ${ }^{4}$ Department \\ of Laboratory Medicine, Seoul \\ National University Bundang \\ Hospital, Seongnam; ${ }^{5}$ Cancer \\ Research Institute, ${ }^{6}$ Department \\ of Laboratory Medicine, ${ }^{7}$ Division \\ of Rheumatology, Department \\ of Internal Medicine, Seoul \\ National University College of \\ Medicine, Seoul, Korea; ${ }^{8}$ Instituto \\ de Parasitología y Biomedicina \\ "López-Neyra", CSIC, Granada, \\ Spain
}

Background/Aims: Myelodysplastic syndrome (MDS) is caused by genetic and epigenetic alteration of hematopoietic precursors and immune dysregulation. Approximately $20 \%$ of patients with MDS develop an autoimmune disease (AID). Here, we investigated whether particular genetic mutations are associated with AID in patients with MDS.

Methods: Eighty-eight genetic mutations associated with myeloid malignancy were sequenced in 73 MDS patients. The association between these mutations and AID was then analyzed.

Results: The median age of the 73 MDS patients was 70 years (interquartile range, 56 to 75 ), and 49 (67.1\%) were male. AID was observed in 16 of 73 patients (21.9\%). Mutations were detected in 57 (78.1\%) patients. The percentage $(68.8 \%$ vs. $80.7 \%$, p $=0.32)$ and the mean number of mutations $(1.8 \pm 1.6$ vs. $2.2 \pm 1.8, p=0.34)$ in MDS patients with or without AID were similar. However, the ten-eleven translocation-2 (TET2) mutation rate was significantly higher in patients with AID than in those without ( $31.3 \%$ vs. $5.3 \%$, respectively; $p=0.001$ ). All TET2 mutations were variants of strong clinical significance.

Conclusions: Mutation of TET2 in patients with MDS may be associated with increased risk of developing AID.

Keywords: Myelodysplastic syndromes; Autoimmune diseases; Mutation

Correspondence to Jin Kyun Park, M.D.

Department of Internal Medicine, Seoul National University College of Medicine, 101 Daehak-ro, Jongno-gu, Seoul, 03080, Korea

Tel: +82-2-2072-4765, Fax: +82-2-762-9662, E-mail: jinkyunpark@gmail.com

Dong-Soon Lee, M.D.

Department of Laboratory Medicine, Seoul National University Hospital, 101 Daehak-ro, Jongno-gu, Seoul 03080, Korea

Tel: +82-2-2072-3986, Fax: +82-2-747-0359, E-mail: soonlee@snu.ac.kr

*These authors contributed equally to this work.
Received: July 5, 2018

Revised : January 6, 2019 Accepted: January 6, 2019 


\section{INTRODUCTION}

Myelodysplastic syndrome (MDS) is a heterogeneous group of clonal hematologic diseases characterized by ineffective hematopoiesis in the bone marrow (BM) and peripheral cytopenia, with the potential to progress to acute myeloid leukemia or fatal BM failure [1]. A key pathophysiologic process in MDS is accumulation of genetic damage. Mutation of genes that regulate critical cellular functions can drive malignant transformation [2]. The resulting unopposed activation and proliferation of immune cells might ultimately disrupt normal immune responses in MDS patients and contribute to development of autoimmune disease (AID). Indeed, approximately $20 \%$ of MDS patients develop AIDs [3], suggesting that MDS-associated AIDs might result from similar genetic changes.

Previously, we demonstrated that certain AIDs in MDS patients are associated with distinctive karyotypic abnormalities and outcomes [4]. While a karyotypic abnormality indicates a radical change in gene dose (with duplication or deletion of large gene segments), genetic modification without karyotypic abnormality can still drive development of MDS. Mutation of these "malignancy-associated genes" contributes to AIDs. One critical mutation may involve proteins that regulate epigenetic modifications, which in turn impact cellular function. For example, mutation of ten-eleven translocation 2 (TET-2) is associated with both MDS and exacerbated inflammatory responses [5].

Here, we investigated whether particular gene mutations frequently associated with myeloid malignancy increase the risk of an AID developing in patients with MDS.

\section{METHODS}

\section{Patients}

A total of 250 patients initially diagnosed with MDS at Seoul National University Hospital from January 2000 to December 2014 were included. Exclusion criteria were as follows: age at the time of diagnosis $<18$ years, initial diagnosis of aplastic anemia or leukemia, and therapy-related MDS. Patients who developed an AID more than 5 years before MDS diagnosis were exclud-

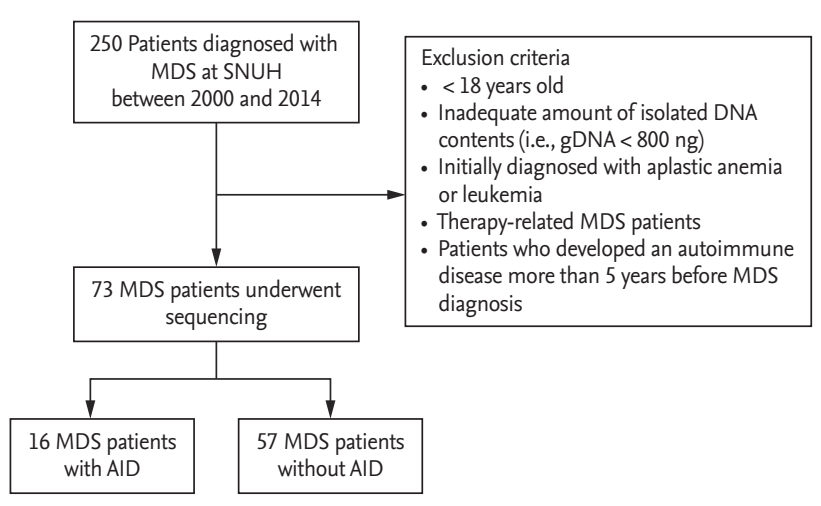

Figure 1. Flow chart of study patients. MDS, myelodysplastic syndrome; SNUH, Seoul National University Hospital; gDNA, genomic DNA; AID, autoimmune disease.

ed. Patients from whom adequate amounts of DNA (i.e., $\geq 800 \mathrm{ng}$ ) could not be extracted were also excluded. A final cohort of 73 treatment-naive patients was included in the analysis (Fig. 1). Laboratory and clinical data were obtained at time of BM sampling for gene sequencing. MDS subtypes were defined according to the World Health Organization classification [6]. The study was approved by the Institutional Review Board of Seoul National University Hospital and conducted in accordance with the Declaration of Helsinki (IRB protocol number: 1604-082-754). Written informed consent was obtained from all patients.

\section{Definition of AID}

Medical records were reviewed to evaluate the presence of an AID. Diagnosis of an AID was based on the 2010 American College of Rheumatology/Annual European Congress of Rheumatology criteria for rheumatoid arthritis (RA) [7], the 1984 modified New York criteria for ankylosing spondylitis [8], and the Bohan and Peter [9] criteria for idiopathic inflammatory myopathies. Autoimmune thyroiditis was defined as abnormal thyroid function in the presence of anti-thyroid antibodies (i.e., anti-thyroid peroxidase antibodies and anti-thyroid stimulating hormone receptor antibodies). Immune thrombocytopenia (ITP) was defined as thrombocytopenia in the presence of antiplatelet antibodies after excluding other causes of thrombocytopenia. Hemolytic anemia (HA) was defined as anemia in the presence of a positive direct Coombs test and laboratory evidence of hemolysis (such as increased lactate dehydrogenase 
levels and decreased haptoglobin levels). Panniculitis, Sweet syndrome, psoriasis, and uveitis were diagnosed clinically by consulting dermatologists and ophthalmologists, respectively.

\section{BM histology}

Wright-stained BM smears and hematoxylin and eosin-stained sections of BM trephine biopsies were reviewed by hematopathologists.

\section{Targeted sequencing of 88 MDS-associated genes}

Eighty-eight genes associated with myeloid malignancy were selected for targeted sequencing (Supplementary Table 1) [10]. To construct a sequencing library, genomic DNA (gDNA) was extracted from BM cells using the QIAamp DNA Blood Mini Kit (Qiagen, Valencia, CA, USA). A total target length of $259 \mathrm{~Kb}$ regions, with paired-ends of $150 \mathrm{bp}$, was sequenced on an Illumina HiSeq 2500 platform (Illumina, San Diego, CA, USA) using the rapid-run sequencing mode. The mean time between MDS diagnosis and gene sequencing was 4.4 days.

\section{Processing of sequencing data and variant calling}

A schematic diagram illustrating the data processing pipeline and the algorithm used for variant calling of somatic mutations is shown in Supplementary Fig. 1. The raw data were mapped to the reference genome (hg19) using the Burrows-Wheeler Aligner (BWA, vo.62). Duplicate PCR reads were removed using Picard 1.98, and variants were called using "UnifiedGenotyper" in GATK 2.7-2. Variants flagged as "LowQual" and low depth (< 10) were excluded. Variants were annotated using ANNOVAR and candidate gene mutations were selected according to the criteria described in the Supplementary Table 1. All synonymous variants were discarded. Missense single nucleotide variants (SNVs) reported in public databases, including single nucleotide polymorphism database (dbSNP), ESP6500, and the 1,000 genomes project, with a frequency of $>0.5 \%$ were filtered as polymorphisms. In addition, an in-house database of Korean polymorphisms, derived from the sequence data of 273 normal Koreans, was used as a private database to filter polymorphisms. SNVs that were found in more than two persons in the Korean database (allele fraction $\geq 0.2 \%$ ) were filtered out. From the filtered SNVs with low depth, SNV variants known from previous MDS studies to be hotspot mutations were rescued [11,12] using the criterion of $>5$ total reads with supporting reads. Mapping errors were confirmed by visual inspection using the IGV browser. Among missense SNVs not reported previously, SNV mutations predicted to be benign by SIFT and Polyphen software were removed.

\section{Statistical analysis}

Continuous variables were expressed as the mean \pm standard deviation or median (interquartile range [IQR]), and categorical variables were expressed as a number (\%). Student's $t$ test and the chi-square test were used to compare continuous or categorical variables between the two groups. A $p$ values $\leq 0.05$ were considered statistically significant. All statistical analyses were performed using SPSS version 23.0 (IBM Co., Armonk, NY, USA).

\section{RESULTS}

\section{Baseline characteristics of MDS patients with or without AID}

The median age of the 73 MDS patients was 70 years (IQR, 56 to 75 ), and 49 patients (67.1\%) were male. The median follow-up duration after MDS diagnosis was 1.8 years (IQR, 0.9 to 3.3). Among the 73 patients, 16 (21.9\%) were diagnosed with an AID: four (25\%) with autoimmune thyroiditis, three (18.8\%) with immune thrombocytopenia, two (12.5\%) with HA, one (6.3\%) with RA, one (6.3\%) with panniculitis, and one $(6.3 \%)$ with idiopathic

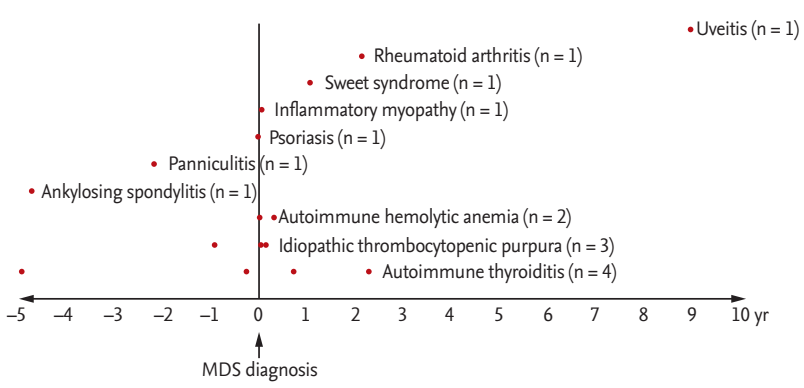

Figure 2. Temporal association between autoimmune disease and myelodysplastic syndrome (MDS) diagnosis. Among the 73 patients, $16(21.9 \%)$ were diagnosed with an AID: four (25\%) autoimmune thyroiditis, three (18.8\%) immune thrombocytopenia, two (12.5\%) hemolytic anemia, one $(6.3 \%)$ RA, one $(6.3 \%)$ panniculitis, one $(6.3 \%)$ idiopathic inflammatory myopathy. 
Table 1. Baseline characteristics in MDS patients with AID or without AID

\begin{tabular}{|c|c|c|c|}
\hline Characteristic & $\operatorname{AID}(n=16)$ & No AID $(\mathrm{n}=57)$ & $p$ value \\
\hline Age at MDS diagnosis, yr & $66.5(56.3-72.5)$ & $70(56-75)$ & 0.66 \\
\hline Female sex & $7(43 \cdot 7)$ & $17(29.8)$ & 0.37 \\
\hline Follow-up duration, yr & $2.1(0.9-4.1)$ & $1.8(0.9-2.9)$ & \\
\hline Autoimmune disease & & NA & \\
\hline Hemolytic anemia & $2(12.5)$ & & \\
\hline ITP & $3(18.8)$ & & \\
\hline Autoimmune thyroiditis & $4(25.1)$ & & \\
\hline Rheumatoid arthritis & $1(6.3)$ & & \\
\hline Ankylosing spondylitis & $1(6.3)$ & & \\
\hline Panniculitis & $1(6.3)$ & & \\
\hline Sweet syndrome & $1(6.3)$ & & \\
\hline Uveitis & $1(6.3)$ & & \\
\hline Psoriasis & $1(6.3)$ & & \\
\hline Inflammatory myositis & $1(6.3)$ & & \\
\hline MDS, subtypes & & & 0.43 \\
\hline RCUD & $3(18.8)$ & $12(21.1)$ & \\
\hline RCMD & $5(31.2)$ & $10(17.5)$ & \\
\hline RAEB & $4(25 \cdot 0)$ & $24(42.1)$ & \\
\hline Others $^{\mathrm{a}}$ & $4(25 \cdot 0)$ & $11(19.3)$ & \\
\hline Karyotypic abnormality & $6(37 \cdot 5)$ & $24(42.1)$ & 0.99 \\
\hline Platelet $\left(\times 10^{3} / \mu \mathrm{L}\right)$ & $126.3 \pm 127.1$ & $115.0 \pm 148.0$ & 0.57 \\
\hline Absolute neutrophil count, $/ \mu \mathrm{L}$ & $1,295 \pm 1,129$ & $1,464 \pm 1,735$ & 0.65 \\
\hline Absolute lymphocyte count, $/ \mu \mathrm{L}$ & $1,180 \pm 860.9$ & $1,021.0 \pm 579.1$ & 0.49 \\
\hline ANA positivity & $1 / 16(6.3)$ & $10 / 56(17.6)$ & 0.82 \\
\hline
\end{tabular}

Values are presented as median (interquartile range), number (\%), or mean \pm SD.

MDS, myelodysplastic syndrome; AID, autoimmune disease; ITP, immune thrombocytopenia; NA, non-applicable; RCUD, refractory cytopenia with unilineage dysplasia; RCMD, refractory cytopenia with multilineage dysplasia; RAEB, refractory anemia with excess blasts; ANA, anti-nuclear antibody.

${ }^{\mathrm{a}}$ Others include refractory anemia with ringed sideroblasts and MDS-unclassifiable.

inflammatory myopathy (Fig. 2). The baseline characteristics of MDS patients with or without an AID are shown in Table 1. The two groups did not differ in terms of sex, age, MDS subtype, and laboratory parameters.

The median time between diagnosis of MDS and AID was 0.03 years (IQR, -0.8 to 1.0). Most AIDs were diagnosed shortly before or after MDS diagnosis. One patient developed uveitis 9 years after MDS diagnosis, and two patients (one RA and one autoimmune thyroiditis) developed AID 2 years after MDS diagnosis. The AIDs were treated with corticosteroid with or without immune suppressants. The case of autoimmune thyroid- itis was treated with methimazole. All patients improved after treatment.

\section{TET2 mutation is associated with increased risk of autoimmunity in MDS patients}

The 88 selected genes sequenced herein are listed in Supplementary Table 1. Fifty-seven of 73 MDS patients (78.1\%) harbored a genetic mutation in at least one of the selected genes. The presence of a mutation $(68.8 \%$ vs. $80.7 \%, p=0.32$ ) and the mean number of mutations ( $1.8 \pm 1.6$ vs. $2.2 \pm 1.8, p=0.34$ ) did not differ between the AID and non-AID groups. Strikingly, the rate of TET2 
mutation was significantly higher in MDS patients with an AID than in those without $31.3 \%[\mathrm{n}=5 / 16]$ vs. $5.3 \%$ [n $=3 / 57]$, respectively; $p=0.001$ ). The patients with TET2 mutation were older than those without (75 [70.8-84] vs. 68 [55.5-73], $p=0.02)$. However, the median age did not differ between the AID and non-AID patients (66.5 years [56.3-72.5] vs. 70 years [56-75], $p=0.66$ ) (Table 1).

Logistic regression analysis revealed that TET2 mutation was associated significantly with an increased risk of AID (odds ratio [OR], 8.18; 95\% confidence interval [CI], 1.70 to 39.38; $p=0.009$ ) (Table 2). All observed TET2 mutations were variants of strong clinical significance, including stop-gain and frameshift deletion and insertion mutations (Table 3).

Table 2. Regression analyses for the development of autoimmune diseases in MDS patients

\begin{tabular}{lcc}
\hline \multirow{2}{*}{ Variable } & \multicolumn{2}{c}{ Univariate } \\
\cline { 2 - 3 } & OR $(95 \% \mathrm{CI})$ & p value \\
\hline Age at MDS diagnosis & $0.99(0.96-1.03)$ & 0.640 \\
Sex, male & $1.83(0.59-5.72)$ & 0.300 \\
IPSS score & $0.95(0.52-1.74)$ & 0.860 \\
TET2 mutation & $8.18(1.70-39.38)$ & 0.009 \\
\hline
\end{tabular}

MDS, myelodysplastic syndrome; OR, odds ratio; CI, confidence interval; IPSS, International Prognostic Scoring System; TET2, ten-eleven translocation 2.

\section{DISCUSSION}

To the best of our knowledge, this study is the first to examine the potential effects of mutations in 88 selected myeloid malignancy-associated genes on development of AID in patients with MDS. Mutation of TET2, an epigenetic regulator, was the single most significant factor that increased the AID risk in MDS patients.

The prevalence of AID in the general population is about $3 \%$ to $5 \%$, whereas that in MDS patients ranges from $10 \%$ to $30 \%$ [13-15]. Consistent with this, 16 (21.9\%) of the patients with MDS in the present study developed an AID. This tight association between MDS and AID indicates that they may share common pathophysiologic mechanisms, including genetic and/or epigenetic modifications. We and others reported that particular karyotypic abnormalities are associated with specific AIDs in patients with MDS. For example, trisomy 8 is associated with Behçet disease in MDS patients [4,16], whereas del (5q) is common in MDS patients with RA [17]. However, karyotypic abnormalities represent a substantial quantitative alteration in whole genome material, and 40.3\% of MDS patients without an abnormal karyotype still develop an AID [4]. Here, we focused on the association between AID and gene mutations that are critical for development of hematologic malignancy [18]. As expected, 57 of 73 MDS patients (78.1\%) harbored mutations in $\geq 1$ of

Table 3. Sequencing of observed TET2-mutations

\begin{tabular}{|c|c|c|c|c|c|c|}
\hline Patient & MDS subtypes & $\begin{array}{l}\text { Classification of } \\
\text { AID }\end{array}$ & $\begin{array}{l}\text { Variant allele } \\
\text { frequency, \% }\end{array}$ & Nucleotide change & $\begin{array}{l}\text { Amino acid } \\
\text { change }\end{array}$ & Type of variants \\
\hline 1 & RCMD & RA & 38.6 & c. $4138 \mathrm{C}>\mathrm{T}$ & p.H138oY & Nonsynonymous \\
\hline 2 & Unclassifiable & AS & 32.0 & c. $4354 \mathrm{C}>\mathrm{T}$ & p.R1452X & Stop gain \\
\hline \multirow[t]{2}{*}{3} & RCUD & Panniculitis & 44.8 & c. $970 \mathrm{C}>\mathrm{T}$ & p.Q324X & Stop gain \\
\hline & & & 40.0 & c.4323-4323delinsTG & p.A1443Cfs & Frameshift insertion \\
\hline \multirow[t]{2}{*}{4} & RCUD & ITP & 32.5 & c. $1342 \mathrm{G}>\mathrm{T}$ & p.E448X & Stop gain \\
\hline & & & 29.1 & c. $4102 \mathrm{~T}>\mathrm{G}$ & p.F1368V & Nonsynonymous \\
\hline 5 & RAEB & Sweet syndrome & 26.9 & c. $2685 \mathrm{delT}$ & p.V895fs & Frameshift deletion \\
\hline 6 & RAEB & None & 29.4 & c. $4827-4828$ insA & - & Frameshift insertion \\
\hline 7 & RAEB & None & 14.8 & c. $A_{5} 686 \mathrm{G}$ & p.R1896G & Nonsynonymous \\
\hline 8 & RCUD & None & $39 \cdot 5$ & c. $\mathrm{C} 1648 \mathrm{~T}$ & p.R550X & Stop gain \\
\hline
\end{tabular}

TET2, ten-eleven translocation 2; MDS, myelodysplastic syndrome; AID, autoimmune disease; RCMD, refractory cytopenia with multilineage dysplasia; RA, rheumatoid arthritis; AS, ankylosing spondylitis; RCUD, refractory cytopenia with unilineage dysplasia; ITP, immune thrombocytopenia; RAEB, refractory anemia with excess blasts. 
88 selected genes associated with hematologic malignancy. Strikingly, only the TET2 mutation was enriched significantly in patients with an AID compared with those without. Subgroup analysis of 43 MDS patients without a karyotypic abnormality revealed that three (30\%) of ten patients with an AID harbored a TET2 mutation compared with three of 33 patients (9\%) without an AID ( $p=$ 0.04) (data not shown). Therefore, alterations at the gene level might be critical for autoimmunity in MDS.

TET2 belongs to the TET family and plays a role in converting 5-methyl-cytosine (5-mC) to 5-hydroxymethyl-cytosine $(5-\mathrm{hmC})$ during DNA demethylation [19]. Accordingly, loss-of-function TET2 mutations lead to decreased TET2 enzymatic activity, which is associated with changes in methylation profiles and epigenetic dysregulation [19]. TET2 deletion is sufficient to initiate myeloid and lymphoid transformation [20]. TET2 controls cytokine production by T cells in AIDs [21]. In addition, TET2 is a crucial negative transcriptional inhibitor of proinflammatory responses, and TET2 depletion contributes to accelerated atherosclerosis via increased inflammasome responses [22]. Accordingly, aberrant methylation of specific gene promoters might be associated with development of hematopoietic malignancy and autoimmunity [20]. In line with this, all TET2 mutations identified herein were variants of strong clinical significance and associated with presumably impaired TET2 function. However, a large prospective study is needed to investigate how TET2 mutation contributes to autoimmunity in MDS.

Previous studies report a strong association between TET2 mutations and aging $[23,24]$. Since TET2 mutations accumulate with age, it might be tempting to speculate that particular AIDs, especially those occurring later in life (e.g., polymyalgia rheumatic and giant cell arteritis [GCA]), might be, at least in part, caused by accumulating TET2 mutations $[25,26]$. A preliminary study revealed that TET2 mutation was not observed in GCA patients without MDS, suggesting that TET2 mutation contributes to development of AID in MDS patients but not in the general population (unpublished data).

The study has several limitations. First, the number of enrolled patients was relatively small, which may affect robust statistical analysis (e.g., correction for multiple comparisons). Second, the temporal relationship between TET2 mutation and AIDs development in patients with MDS could not be investigated precisely since the exact time of TET2 mutation could not be determined. Third, AID cases diagnosed within 5 years before MDS diagnosis were included, since chronic smoldering cases of MDS might remain undiagnosed for many years until emergence of obvious clinical symptoms. However, it is possible that those cases might not be related to MDS. Fourth, ITP in three patients was defined as isolated thrombocytopenia in the presence of antiplatelet antibodies after excluding other causes of thrombocytopenia. However, it is difficult to differentiate ITP from dysplastic megakaryopoiesis with a high degree of certainty. In addition, one patient with autoimmune thyroiditis after allogenic peripheral blood stem cell transplantation was included as an MDS-related AID. Since hematopoietic cell transplantation might clear MDS clones that might induce Grave's disease, Graves' disease occurring after hematopoietic cell transplantation might not be related to MDS; rather, it could be a manifestation of graft versus host disease. Finally, it would be of particular interest to investigate whether disappearance of clones with TET2 mutations after treatment of MDS (e.g., with BM transplantation) is associated with improvement of autoimmune phenomena. Further studies are warranted to validate the current finding in independent cohorts.

In conclusion, this study is the first to show that mutation of TET2 may be associated with a significant increase in the risk of AID in patients with MDS. Large prospective and validation studies are needed to identify more precise relationships and underlying mechanisms.

\section{KEY MESSAGE}

1. Autoimmunity is common in myelodysplastic syndrome (MDS).

2. Mutation of ten-eleven translocation 2 (TET-2), an epigenetic regulator, is associated significantly with increased risk of autoimmunity in MDS.

3. Particular genetic alterations might contribute to both autoimmunity and malignancy in MDS. 


\section{Conflict of interest}

No potential conflict of interest relevant to this article was reported.

\section{Acknowledgments}

We thank Professor Eun Bong Lee for critical review of the manuscript. This research was supported by Basic Science Research Program through the National Research Foundation of Korea (NRF), funded by the Ministry of Science, ICT and Future Planning (NRF2017R1A2A1A17069780).

\section{REFERENCES}

1. Glenthoj A, Orskov AD, Hansen JW, Hadrup SR, O'Connell C, Gronbaek K. Immune mechanisms in myelodysplastic syndrome. Int J Mol Sci 2016;17:E944.

2. Yogarajah M, Tefferi A. Leukemic transformation in myeloproliferative neoplasms: a literature review on risk, characteristics, and outcome. Mayo Clin Proc 2017;92:1118-1128.

3. Giannouli S, Voulgarelis M, Zintzaras E, Tzioufas AG, Moutsopoulos HM. Autoimmune phenomena in myelodysplastic syndromes: a 4-yr prospective study. Rheumatology (Oxford) 2004;43:626-632.

4. Lee SJ, Park JK, Lee EY, et al. Certain autoimmune manifestations are associated with distinctive karyotypes and outcomes in patients with myelodysplastic syndrome: a retrospective cohort study. Medicine (Baltimore) 2016;95:e3091.

5. Ades L, Itzykson R, Fenaux P. Myelodysplastic syndromes. Lancet 2014;383:2239-2252.

6. Vardiman JW, Thiele J, Arber DA, et al. The 2008 revision of the World Health Organization (WHO) classification of myeloid neoplasms and acute leukemia: rationale and important changes. Blood 2009;114:937-951.

7. Aletaha D, Neogi T, Silman AJ, et al. 2010 Rheumatoid arthritis classification criteria: an American College of Rheumatology/European League Against Rheumatism collaborative initiative. Arthritis Rheum 2010;62:25692581 .

8. van der Linden S, Valkenburg HA, Cats A. Evaluation of diagnostic criteria for ankylosing spondylitis. A proposal for modification of the New York criteria. Arthritis Rheum 1984;27:361-368.
9. Bohan A, Peter JB. Polymyositis and dermatomyositis (first of two parts). N Engl J Med 1975;292:344-347.

10. Mughal TI, Cross NC, Padron E, et al. An International MDS/MPN Working Group's perspective and recommendations on molecular pathogenesis, diagnosis and clinical characterization of myelodysplastic/myeloproliferative neoplasms. Haematologica 2015;100:1117-1130.

11. Papaemmanuil E, Gerstung M, Malcovati L, et al. Clinical and biological implications of driver mutations in myelodysplastic syndromes. Blood 2013;122:3616-3627.

12. Haferlach T, Nagata Y, Grossmann V, et al. Landscape of genetic lesions in 944 patients with myelodysplastic syndromes. Leukemia 2014;28:241-247.

13. Braun T, Fenaux P. Myelodysplastic syndromes (MDS) and autoimmune disorders (AD): cause or consequence? Best Pract Res Clin Haematol 2013;26:327-336.

14. Cooper GS, Bynum ML, Somers EC. Recent insights in the epidemiology of autoimmune diseases: improved prevalence estimates and understanding of clustering of diseases. J Autoimmun 2009;33:197-207.

15. Komrokji RS, Kulasekararaj A, Al Ali NH, et al. Autoimmune diseases and myelodysplastic syndromes. Am J Hematol 2016;91:E280-E283.

16. Esatoglu SN, Hatemi G, Salihoglu A, Hatemi I, Soysal T, Celik AF. A reappraisal of the association between Behçet's disease, myelodysplastic syndrome and the presence of trisomy 8: a systematic literature review. Clin Exp Rheumatol 2015;33(6 Suppl 94):S145-S151.

17. Giagounidis AA, Haase S, Germing U, Heinsch M, Aul C. Autoimmune disorders in two patients with myelodysplastic syndrome and $5 q$ deletion. Acta Haematol 2005;113:146-149.

18. Vainchenker W, Kralovics R. Genetic basis and molecular pathophysiology of classical myeloproliferative neoplasms. Blood 2017;129:667-679.

19. Solary E, Bernard OA, Tefferi A, Fuks F, Vainchenker W. The ten-eleven translocation-2 (TET2) gene in hematopoiesis and hematopoietic diseases. Leukemia 2014;28:485-496.

20. Ko M, Bandukwala HS, An J, et al. Ten-eleven-translocation 2 (TET2) negatively regulates homeostasis and differentiation of hematopoietic stem cells in mice. Proc Natl Acad Sci U S A 2011;108:14566-14571.

21. Ichiyama K, Chen T, Wang X, et al. The methylcytosine dioxygenase Tet2 promotes DNA demethylation and activation of cytokine gene expression in T cells. Immunity 
2015;42:613-626.

22. Fuster JJ, MacLauchlan S, Zuriaga MA, et al. Clonal hematopoiesis associated with TET2 deficiency accelerates atherosclerosis development in mice. Science 2017;355:842847.

23. Langemeijer SM, Jansen JH, Hooijer J, et al. TET2 mutations in childhood leukemia. Leukemia 2011;25:189-192.

24. Metzeler KH, Maharry K, Radmacher MD, et al. TET2 mutations improve the new European LeukemiaNet risk classification of acute myeloid leukemia: a Cancer and Leukemia Group B study. J Clin Oncol 2011;29:1373-1381.

25. Busque L, Patel JP, Figueroa ME, et al. Recurrent somatic TET2 mutations in normal elderly individuals with clonal hematopoiesis. Nat Genet 2012;44:1179-1181.

26. Mohan SV, Liao YJ, Kim JW, Goronzy JJ, Weyand CM. Giant cell arteritis: immune and vascular aging as disease risk factors. Arthritis Res Ther 2011;13:231. 
Supplementary Table 1. Genetic mutational profiles of MDS patients with or without AID

\begin{tabular}{|c|c|c|c|}
\hline Variable & $\operatorname{AID}(n=16)$ & No AID $(n=57)$ & $p$ value \\
\hline Presence of genetic mutation & $11(68.8)$ & $46(80.7)$ & 0.32 \\
\hline No. of mutated genes & & & 0.32 \\
\hline o & $5(31.3)$ & $11(19 \cdot 3)$ & \\
\hline 1 & $3(18.8)$ & $13(22.8)$ & \\
\hline 2 & $3(18.8)$ & $10(17.5)$ & \\
\hline 3 & $1(6.3)$ & $12(21.1)$ & \\
\hline 4 & $4(25.0)$ & $4(7.0)$ & \\
\hline 5 & $\circ$ & $2(3.5)$ & \\
\hline 6 & $\circ$ & $4(7.0)$ & \\
\hline 7 & $\circ$ & $1(1.8)$ & \\
\hline \multicolumn{4}{|l|}{ Classifier of mutated genes } \\
\hline \multicolumn{4}{|l|}{ Chromatin modification } \\
\hline$A S X L_{1}$ & $3(18.8)$ & $13(22.9)$ & 0.84 \\
\hline $\mathrm{EZH} 2$ & $1(6.3)$ & $4(7.0)$ & 1.0 \\
\hline \multicolumn{4}{|l|}{ Splicing } \\
\hline $\mathrm{PRPF}_{4} \mathrm{OB}$ & $\circ$ & $1(1.8)$ & 1.0 \\
\hline$S_{5} B_{1}$ & $2(12.5)$ & $4(7.0)$ & 0.61 \\
\hline SRSF2 & $2(12.5)$ & $3(5.3)$ & 0.3 \\
\hline$U_{2} A F_{1}$ & $\circ$ & $9(15.8)$ & 0.19 \\
\hline $\mathrm{U}_{2} \mathrm{AF}_{2}$ & $\circ$ & $1(1.8)$ & 1.0 \\
\hline ZRSR2 & $1(6.3)$ & $2(3.5)$ & 0.53 \\
\hline \multicolumn{4}{|l|}{ DNA methylation } \\
\hline $\mathrm{DNMT}_{3} \mathrm{~A}$ & $1(6.3)$ & $5(8.8)$ & 0.86 \\
\hline $\mathrm{IDH} 2$ & $\circ$ & $3(5.3)$ & 1.0 \\
\hline TET $_{2}$ & $5(31.3)$ & $3(5.3)$ & 0.001 \\
\hline \multicolumn{4}{|l|}{ Transcription } \\
\hline BCOR & o & $4(7.0)$ & 0.57 \\
\hline $\mathrm{BRD}_{4}$ & 0 & $1(1.8)$ & 1.0 \\
\hline CEBPA & o & $3(5.3)$ & 1.0 \\
\hline EGR2 & o & $1(1.8)$ & 1.0 \\
\hline ETV6 & o & $1(1.8)$ & 1.0 \\
\hline GATA1 & o & $1(1.8)$ & 1.0 \\
\hline GATA2 & ○ & $1(1.8)$ & 1.0 \\
\hline$I_{K Z F}$ & o & $1(1.8)$ & 1.0 \\
\hline NPM1 & o & $2(3.5)$ & 1.0 \\
\hline$R U N X_{1}$ & $1(6.3)$ & $4(7.0)$ & 1.0 \\
\hline${ }^{T P_{53}}$ & $2(12.5)$ & $12(21.1)$ & 0.31 \\
\hline WT1 & $1(6.3)$ & $2(3.5)$ & 0.53 \\
\hline \multicolumn{4}{|l|}{ Receptor kinase } \\
\hline $\mathrm{BIRC}_{3}$ & o & $1(1.8)$ & 1.0 \\
\hline $\mathrm{CSF}_{3} \mathrm{R}$ & o & $3(5.3)$ & 1.0 \\
\hline JAK2 & o & $3(5 \cdot 3)$ & 1.0 \\
\hline
\end{tabular}




\section{Supplementary Table 1. Continued}

\begin{tabular}{|c|c|c|c|}
\hline Variable & $\operatorname{AID}(n=16)$ & No AID $(n=57)$ & $p$ value \\
\hline MPL & 0 & $2(3 \cdot 5)$ & 1.0 \\
\hline $\mathrm{NOTCH}$ & 0 & $4(7.0)$ & 0.57 \\
\hline \multicolumn{4}{|l|}{ RAS pathway } \\
\hline$N F_{1}$ & 0 & $2(3 \cdot 5)$ & 1.0 \\
\hline NRAS & o & $2(3.5)$ & 1.0 \\
\hline PTPNII & 0 & $1(1.8)$ & 1.0 \\
\hline \multicolumn{4}{|l|}{ Signaling } \\
\hline $\mathrm{PRKD}_{3}$ & $1(6.3)$ & 0 & 0.22 \\
\hline $\mathrm{SH}_{2} \mathrm{~B}_{3}$ & 0 & $1(1.8)$ & 1.0 \\
\hline \multicolumn{4}{|c|}{ Cell cycle/DNA repair } \\
\hline$R B_{1}$ & $1(6.3)$ & $1(1.8)$ & 0.39 \\
\hline ATM & 0 & $3(5 \cdot 3)$ & 1.0 \\
\hline$B A R D_{1}$ & 0 & $1(1.8)$ & 1.0 \\
\hline \multicolumn{4}{|l|}{ Cohesin } \\
\hline$S M C_{1} A$ & 0 & $1(1.8)$ & 1.0 \\
\hline$S M C_{3}$ & 0 & $1(1.8)$ & 1.0 \\
\hline STAG2 & $1(6.3)$ & $3(5 \cdot 3)$ & 1.0 \\
\hline \multicolumn{4}{|l|}{ Others } \\
\hline$D_{I} S_{3}$ & 0 & $1(1.8)$ & 1.0 \\
\hline $\mathrm{FAT}_{4}$ & 0 & $3(5 \cdot 3)$ & 1.0 \\
\hline $\mathrm{LAMB}_{4}$ & 0 & $2(3 \cdot 5)$ & 1.0 \\
\hline$L R P_{1} B$ & 0 & $2(1.6)$ & \\
\hline $\mathrm{PLEKHG}_{5}$ & $1(6.3)$ & $3(5 \cdot 3)$ & 1.0 \\
\hline POLG & $1(6.3)$ & $1(1.8)$ & 0.39 \\
\hline RIPKI & 0 & $1(1.8)$ & 1.0 \\
\hline SCRIB & 0 & $1(1.8)$ & 1.0 \\
\hline SETBP1 & 0 & $1(1.8)$ & 1.0 \\
\hline TGM7 & o & $1(1.8)$ & 1.0 \\
\hline
\end{tabular}

Values are presented as number (\%). No mutation was found 39 of 88 genes; ATRX, SF1, SF3A1, IDH1, BRD2, PHF6, TCF12, CSF1 R, FBXW7, FLT 3 , KIT, BRAF, CBL, KRAS, ITPKB, MYDSS, CCND1, CDKN2A, BRCC 3 , RAD21, CALR, CARD6, CHD2, DAP3, $\mathrm{DDX}_{3} \mathrm{X}, \mathrm{EEF}_{1} \mathrm{E}_{1}, \mathrm{FAM}_{4} 6 \mathrm{C}, \mathrm{HIST} \mathrm{H}_{1} \mathrm{E}, \mathrm{KIAA0355}$, KLHL6, MED12, MAPK1, NFKBIE, POT1, PTEN, SAMND1, SMARCA2, XPO1, ZMYM3.

MDS, myelodysplastic syndrome; AID, autoimmune disease. 


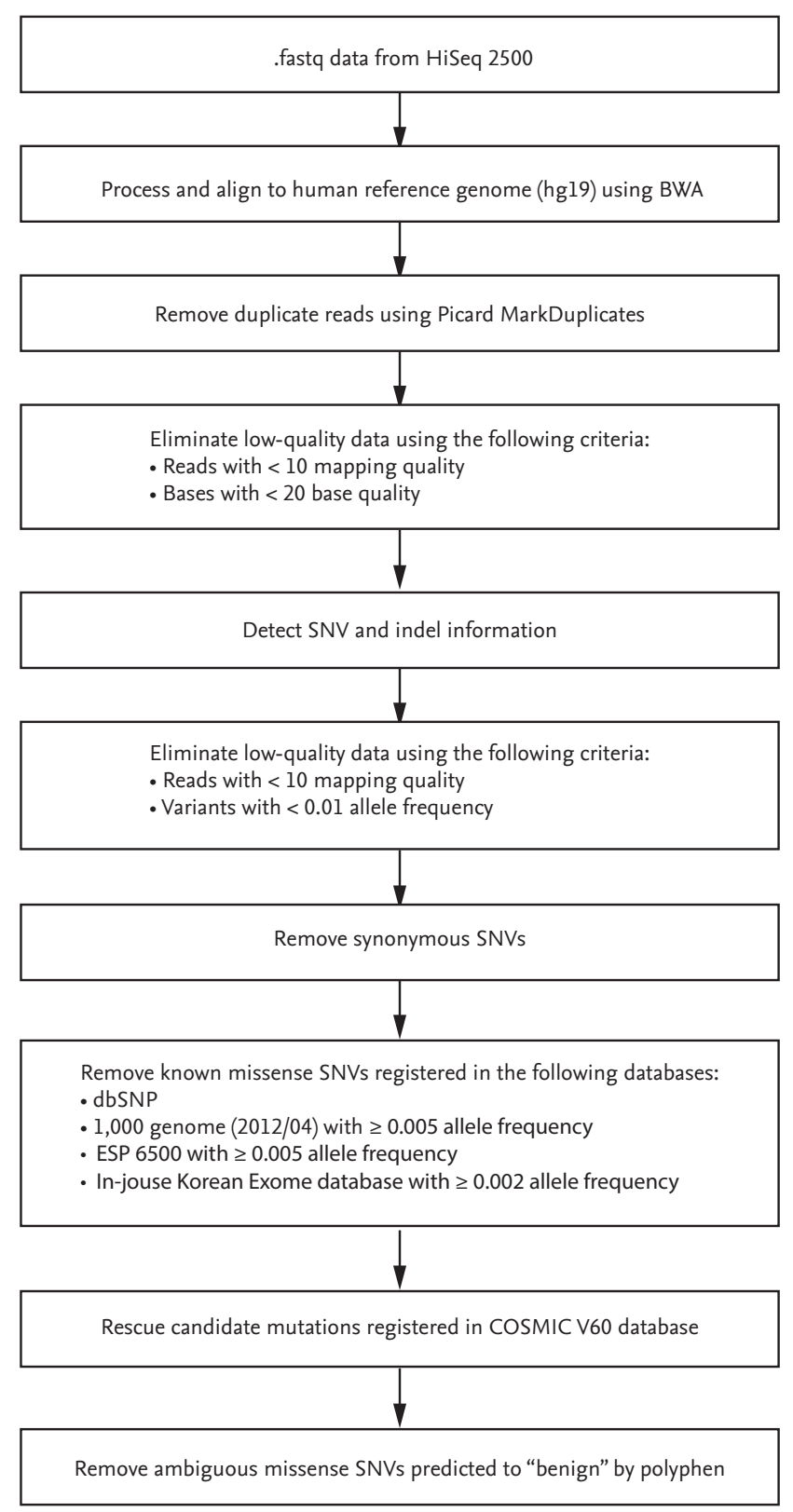

Supplementary Figure 1. Sequencing data processing and variant calling process. BWA, Burrows-Wheeler-Aligner; SNV, single nucleotide variants; dbSNP, single nucleotide polymorphism database; ESP, exome sequencing project; COSMIC, Catalogue Of Somatic Mutations In Cancer. 